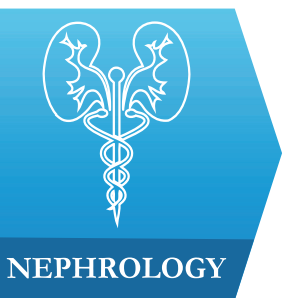

1) Department of Medicine and Division of Nephrology, Pt. B.D. Sharma University of Health Sciences, Rohtak (Haryana), India

2) Department of Medicine and Division of Nephrology, Pt. B.D. Sharma University of Health Sciences, Rohtak (Haryana), India

3) Department of Medicine, Pt. B.D. Sharma University of Health Sciences, Rohtak (Haryana), India

4) Department of Pathology, Pt. B.D. Sharma University of Health Sciences, Rohtak (Haryana), India

\section{Evaluation of thyroid dysfunction in patients with nephrotic syndrome}

Deepak Jain ${ }^{1}$, Hari Krishan Aggarwal², Pavan Kumar YM³ ${ }^{3}$ Promil Jain ${ }^{4}$

\begin{abstract}
Background. Thyroid and kidney are interdependent on each other in many ways for optimal functioning of either organs. Proteinuria causes urinary loss of thyroid hormones and thyroid binding globulins in substantial amount resulting in subclinical/ overt hypothyroidism. Autoimmunity, which can attack both the organs simultaneously, may also contribute considerably to the abnormal functioning of both organs.
\end{abstract}

Aims and objectives. To study the effect of proteinuria on the thyroid function and its association with autoimmunity.

Methods. The study was carried out on a total number of 60 patients with nephrotic range proteinuria attending the kidney and dialysis clinic PGIMS, Rohtak, India. Thyroid profile and baseline investigations along with Anti-TPO antibodies and renal biopsy were carried out on each patient. Patients were allocated to 2 groups based on Anti TPO antibody results: group A comprising 25 Anti-TPO Ab positive patients and group B comprising 35 Anti-TPO Ab negative patients.

Results. Group A patients with Anti TPO antibody positivity had more elevated TSH levels $(\mathrm{p}<0.0001)$, proteinuria $(\mathrm{p}=0.0011)$ and serum creatinine $(\mathrm{p}=0.0137)$ as compared to group B patients. Group A patients had more diminished eGFR $(\mathrm{p}=0.0127)$ and serum albumin $(\mathrm{p}=0.0056)$ than patients in group B. TSH levels were positively correlated with proteinuria $\mathrm{r}=0.55(\mathrm{p}<0.0001,95 \%$ CI 0.35 to 0.70$)$ and serum creatinine levels $\mathrm{r}=0.56$ $(\mathrm{p}<0.0001,95 \%$ CI 0.36 to 0.71$)$. TSH levels were negatively correlated with serum albumin levels $\mathrm{r}=-0.52(\mathrm{p}<0.0001$, CI -0.68 to -0.31$)$ and glomerular filtration rate $\mathrm{r}=-$ 0.54 ( $\mathrm{p}<0.0001, \mathrm{CI}-0.69$ to -0.33 ). On histopathology, membranous nephropathy -29 out of 60 patients, $48 \%$ - was the most common finding in both the groups.

Conclusions. Nephrotic range proteinuria leading to thyroid dysfunction is a common entity but the association with autoimmunity causes an exaggerated effect on both these organs. Our study established a significant correlation between thyroid autoimmunity and nephrotic syndrome. Thus, a high index of suspicion should be kept in all patients with nephrotic syndrome to look for any associated antibodies against thyroid antigens. Autoimmunity causes more proteinuria and more elevations of thyrotropin leading to clinical hypothyroidism; however, the occurrence of overt hypothyroidism necessitating treatment needs further study.

Keywords: proteinuria, thyroid dysfunction, hypothyroidism

The interaction between kidney and thyroid hormones is an important factor in the normal functioning of both the vital organs. Thyroid hormones, on the one hand, plays a major role in the early development of kidney structurally and thereafter in the regulation of major glomerular and tubular functions as well as water and electrolyte balance. Kidneys, on the other hand, are important in the metabolism and elimination of thyroid hormones [1]. Thus, defect in functioning of either organs are expected to have consequences on both systems. Renal diseases are widely characterized by the presence of proteinuria. Although albumin constitutes the major fraction of protein excreted due to selective nature of proteinuria, many other important globulins, hormones and hormone binding proteins are also excreted in significant amount which leads to clinical implications other than pertaining to renal dysfunction itself. Urinary losses 
of thyroxine and thyroid binding globulins (TBG) is one such important concern. Although in early stages no metabolic consequences are expected since levels of free Triiodothyronine (fT3) and free Thyroxine (fT4) remain normal, prolonged excretions of TBG can reduce levels of free thyroid hormones, which leads to rise in thyroid stimulating hormone (TSH) levels as the thyroid gland starts to over-function in order to maintain the steady hormone levels [2]. Although most patients are able to maintain a clinical euthyroid state with these changes, patients with low thyroid reserves can develop overt hypothyroidism. Autoimmunity, which can attack both organs simultaneously, can also contribute considerably to abnormal functioning of both organs $[3,4]$. Regardless of the cause, simultaneous dysfunction of both organs can substantially contribute to the patient morbidity. Thus, a thorough knowledge of exact pathophysiology is required to plan an effective treatment protocol in these patients. Although there are a few studies and case reports of hypothyroidism with nephrotic syndrome, substantial data are still lacking to study the thyroid hormone status and its clinical relevance in patients with nephrotic syndrome.

Nephrotic syndrome is one of the best-known presentations of adult kidney disease. It can be caused by a wide range of primary (idiopathic) and secondary glomerular diseases. It is, in fact, an important manifestation of kidney disease [5]. Nephrotic syndrome, characterized mainly by urinary protein loss, also results in urinary losses of binding proteins, such as thyroxine binding globulin (TBG), transthyretin or prealbumin, albumin, and thyroid hormones bound to them. Hypothyroidism-associated nephrotic syndrome seems to be related more to the decline in thyroid hormone levels rather than to thyroid autoimmunity. In the past, there have been a number of case reports linking hypothyroidism and nephrotic syndrome, but detailed studies are still lacking. Therefore, this study was planned to know the factors affecting the association between the nephrotic syndrome and thyroid dysfunction.

\section{Material and method}

This observational study was carried out on adult patients with nephrotic syndrome enrolled in the kidney \& dialysis clinic of PGIMS, Rohtak, India. Patients aged $<18$ years, hypothalamic-pituitary axis dysfunction, pituitary tumors, absent thyroid gland, thyroid gland tumors before the onset of nephrotic syndrome were excluded from the study. Written informed consent was taken from all the participants. The study was approved by the ethical committee of Pt. B. D. Sharma University of Health Sciences, Rohtak, India. Those who denied participating in the study were also excluded.

After a detailed history and clinical examination, biochemical and other necessary investigations were done to fulfill the inclusion criteria. Serum freeT3 (fT3), freeT4 (fT4), Thyroid Stimulating Hormone (TSH), Anti-Thyroid
Peroxidase (Anti-TPO) and Anti-thyroglobulin (Anti-Tg) were measured in all the study subjects. Normal level was considered as Serum fT3 $=1.71-3.71 \mathrm{pg} / \mathrm{ml}$, fT4 $=0.8-1.7 \mathrm{ng} /$ $\mathrm{dl}, \mathrm{TSH}=0.3-5 \mathrm{mIU} / \mathrm{L}$, anti-TPO $\mathrm{Ab}<60 \mathrm{IU} / \mathrm{ml}$ and anti-Tg $<60 \mathrm{U} / \mathrm{ml}$. Serum fT3, fT4, TSH, Anti-Tg and Anti-TPO were measured quantitatively using Chemiluminescent Immunoassay (CLIA). This is an in-vitro diagnostic sandwich immunoassay built on Streptavidin-Biotin based plates detected using Semi-automated analyzer. Same CLIA kits were used throughout the study period. eGFR was calculated using the MDRD formula. Proteinuria was measured using standard 24-hr urine collection. Renal biopsy was done in all study subjects to confirm the type of glomerulonephritis by histopathological and immunofluorescence techniques. Ultrasound examination of the thyroid gland was performed in all patients, using a high frequency transducer in supine position with hyperextended neck both in transverse and longitudinal planes. Renal biopsy was obtained in all patients under strict aseptic precautions and after ruling out coagulopathy, using real-time ultrasound guidance and disposable core biopsy needle; percutaneous renal biopsy was performed with patient in prone position. Specimen was sent for both light microscopy (placed in formalin) and immunofluorescence examination (placed in normal saline and quickly freezed).

About 400 patients were screened during a period of 12 months; after due consideration of inclusion and exclusion criteria, 60 patients were enrolled for the study. Further, depending upon anti-TPO antibody positivity, patients were divided into 2 groups. Group A comprised 25 patients positive for anti-TPO antibody and group B comprised 35 patients who were negative for anti-TPO antibody.

IBM SPSS Statistics software version 20 was used for the statistical analysis. Continuous variables [fT3, $\mathrm{TSH}$, proteinuria, eGFR] were mentioned as mean $\pm \mathrm{SD}$ and categorical variables (types of glomerulonephritis) were mentioned as percentages. Continuous variables between 2 groups were analyzed using independent t-test after $\log 10$ transformation (to reduce the skewness). The correlation between two independent variables of the study population was estimated using the Pearson's correlation coefficient (r). A p-value of $<0.05$ is considered statistically significant.

\section{Results}

Sixty patients, fulfilling the criteria of concomitant nephrotic syndrome and thyroid dysfunction in the form of clinical or subclinical hypothyroidism, were included in the study. The mean age of study population was $36.74 \pm 10.63$ years. All patients had nephrotic range proteinuria with mean 24-hr proteinuria values of $4.61 \pm 1.37$ gm/day. Patients had decreased mean serum albumin levels with a value of $2.96 \pm 0.69 \mathrm{mg} / \mathrm{dl}$. As all patients had either subclinical or clinical hypothyroidism the mean TSH values was $26.73 \pm 27.68 \mathrm{mIU} / \mathrm{L}$. The mean fT3 and fT4 values were $2.46 \pm 0.69 \mathrm{pg} / \mathrm{ml}$ and $1.21 \pm 0.49 \mathrm{ng} / \mathrm{dl}$. Anti 
TPO antibodies were positive in $42 \%$ of subjects. The patients had mean Anti TPO Ab levels of 191.96 \pm 257.77 $\mathrm{IU} / \mathrm{ml}$. Anti-Tg antibodies were positive in $35 \%$ of patients with mean levels of $55.2 \pm 31.57 \mathrm{U} / \mathrm{ml}$. Of the 60 patients, 2 patients who tested negative for Anti-TPO Ab were positive for Anti-Tg antibodies.

Group A patients with positive Anti-TPO antibodies had higher TSH levels as compared to group B patients who were negative for Anti-TPO antibodies [Figure 1] and the difference was statistically significant $(\mathrm{p}<0.05)$.

The fT3 and fT4 levels were lower in group A patients as compared to group B patients. Group A patients had lower serum albumin as compared to group B patients and the difference was statistically significant $(p<0.05)$. Group A patients had higher serum creatinine values as compared to group B patients and the difference was statistically significant $(\mathrm{p}<0.05)$. Group A patients had higher 24-hr proteinuria as compared to group B patients and the difference was statistically significant $(\mathrm{p}<0.05)$.
Group A patients had lower eGFR values as compared to group B patients and the difference was statistically significant $(p<0.05)$ (Table I). Lower eGFR values in group A patients could be explained by the underlying autoimmunity in them.

In our study, $33 \%$ patients had subclinical hypothyroidism. TSH levels were correlated with various parameters using Pearson correlation coefficient analysis. TSH levels correlated positively with proteinuria and the correlation coefficient ' $\mathrm{r}$ ' was 0.55 ( $\mathrm{p}<0.0001,95 \%$ CI 0.35 to 0.70 ) (Figure 2). TSH levels correlated positively with serum creatinine levels and the correlation coefficient ' $\mathrm{r}$ ' was 0.56 ( $<<0.0001,95 \%$ CI 0.36 to 0.71$)$ (Figure 3). TSH levels correlated negatively with serum albumin levels and the correlation coefficient ' $\mathrm{r}$ ' was -0.52 ( $\mathrm{p}<0.0001, \mathrm{CI}-0.68$ to -0.31) (Figure 4). TSH levels correlated negatively with glomerular filtration rate and the correlation coefficient ' $r$ ' was $-0.54(p<0.0001, C I-0.69$ to -0.33$)$ (Figure 5).

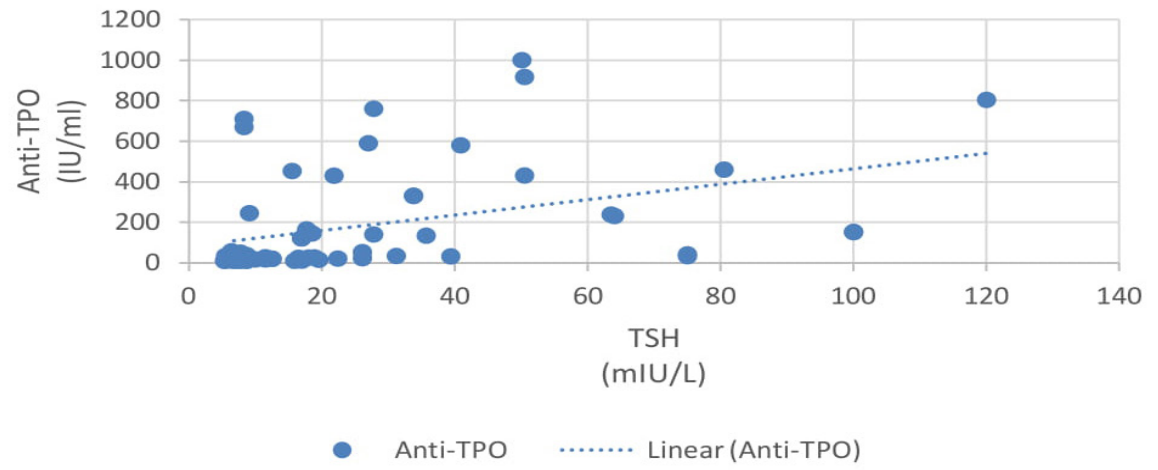

Figure1. Correlation of Anti-TPO Ab with TSH.

Table I. Comparison of group A vs. group B.

\begin{tabular}{|c|c|c|c|}
\hline & $\begin{array}{c}\text { Anti-TPO antibodies positive } \\
\text { Group A } \\
25(41.7 \%)\end{array}$ & $\begin{array}{c}\text { Anti-TPO antibodies negative } \\
\text { Group B } \\
35(58.3 \%)\end{array}$ & P value \\
\hline Age & $36.65 \pm 11.1$ & $36.22 \pm 10.32$ & $>0.2$ \\
\hline BMI & $21.4 \pm 1.4$ & $21.8 \pm 1.6$ & $>0.2$ \\
\hline fT3 $(\mathrm{pg} / \mathrm{ml})$ & $2.08 \pm 0.77$ & $2.73 \pm 0.46$ & $<0.05$ \\
\hline fT4 (ng/dl) & $1.19 \pm 0.71$ & $1.22 \pm 0.23$ & $>0.2$ \\
\hline TSH (mIU/L) & $40.76 \pm 33.04$ & $16.69 \pm 16.39$ & $<0.05$ \\
\hline Serum albumin $(\mathrm{gm} / \mathrm{dl})$ & $2.66 \pm 0.48$ & $3.17 \pm 0.73$ & $<0.05$ \\
\hline Serum creatinine $(\mathrm{mg} / \mathrm{dl})$ & $1.51 \pm 0.69$ & $1.13 \pm 0.52$ & $<0.05$ \\
\hline Proteinuria (gm/day) & $4.01 \pm 1.86$ & $2.71 \pm 1.79$ & $<0.05$ \\
\hline eGFR (ml/min/1.73m2) & $58.06 \pm 24.07$ & $77.17 \pm 27.89$ & $<0.05$ \\
\hline Anti TPO Antibodies (IU/ml) & $412.40 \pm 267.41$ & $24.99 \pm 13.78$ & $<0.05$ \\
\hline Anti- Tg (U/ml) & $55.21 \pm 32.3$ & $55.12 \pm 30.6$ & $>0.2$ \\
\hline
\end{tabular}




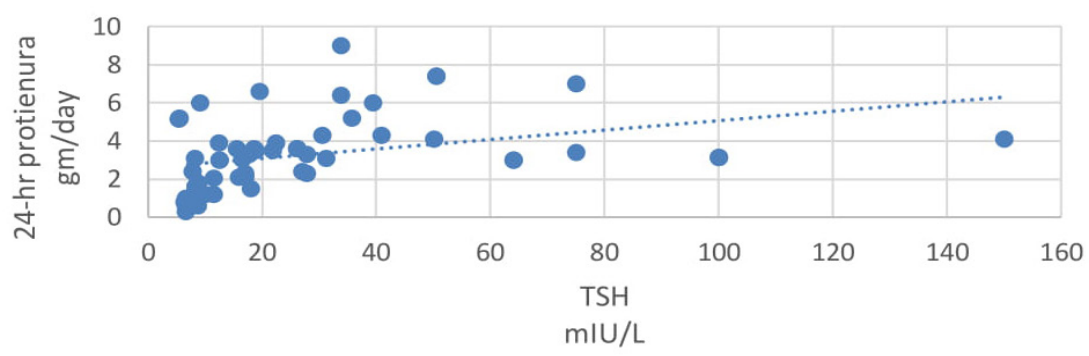

- $24 \mathrm{Hr}$ proteinuria Linear (24 Hr proteinuria)

Figure 2. Correlation of 24-hr proteinuria with TSH.

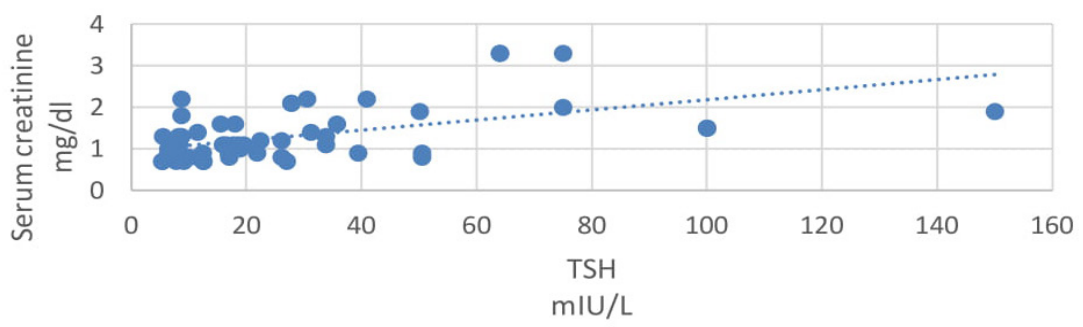

- Serum creatinine Linear (Serum creatinine)

Figure 3. Correlation of serum creatinine with TSH.

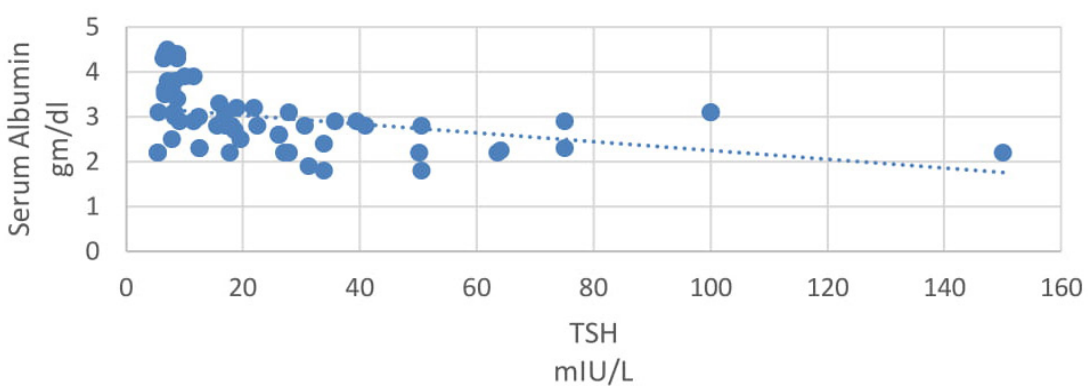

- Serum Albumin $\quad$........ Linear (Serum Albumin)

Figure 4. Correlation of serum albumin with TSH.

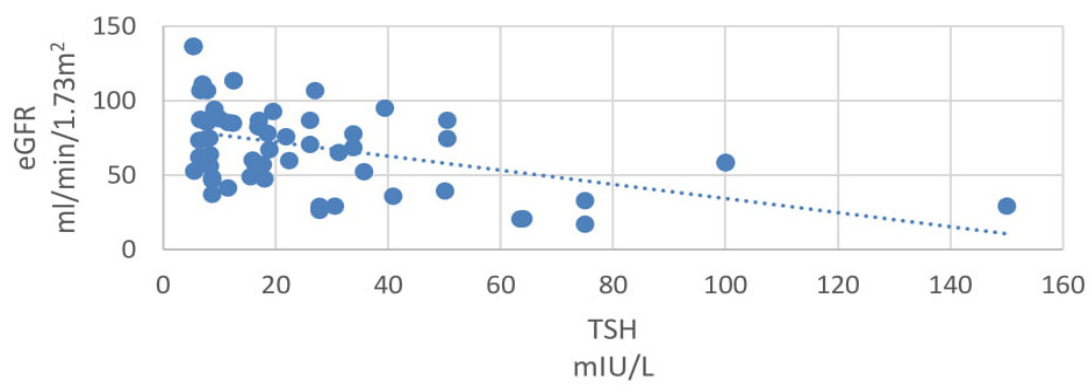

- eGFR …... Linear (eGFR)

Figure 5. Correlation of eGFR with TSH. 
On histopathological examination of renal biopsy, the most common finding was membranous glomerulopathy $(48 \%)$ in both the groups (Figure 6). Other findings included focal segmental glomerulonephritis (15\%), minimal change glomerulonephritis (12\%), lupus nephritis (10\%), mesangioproliferative glomerulonephritis (7\%), membranoproliferative glomerulonephritis (5\%), and IgA nephropathy (3\%) (Table II).

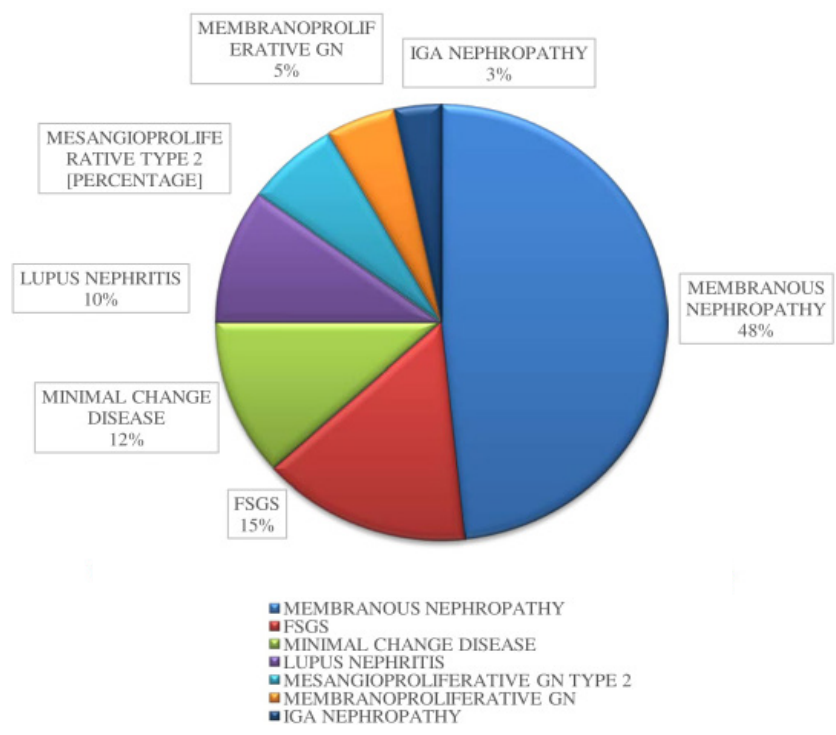

Figure 6. Various types of glomerulonephritis in this study.

Table II. Types of glomerulonephritis in the study groups.

\begin{tabular}{|l|c|c|c|}
\hline Type of glomerulonephritis & $\begin{array}{c}\text { Total } \\
\mathbf{6 0}\end{array}$ & $\begin{array}{c}\text { Group A } \\
\mathbf{2 5}(\mathbf{4 1 . 7 \% )})\end{array}$ & $\begin{array}{c}\text { Group B } \\
\mathbf{3 5}(\mathbf{5 8 . 3} \%)\end{array}$ \\
\hline Membranous GN & $29(48 \%)$ & 8 & 21 \\
FSGS & $9(15 \%)$ & 8 & 1 \\
Minimal change & $7(12 \%)$ & 3 & 4 \\
Lupus nephritis & $6(10 \%)$ & 4 & 2 \\
Mesangioproliferative type 2 & $4(7 \%)$ & 1 & 3 \\
Membranoproliferative & $3(5 \%)$ & 1 & 2 \\
IgA Nephropathy & $2(3 \%)$ & 0 & 2
\end{tabular}

\section{Discussion}

Clinical hypothyroidism was defined as elevated TSH levels $(>10 \mathrm{mIU} / \mathrm{L})$ along with low or normal levels of serum T4. Subclinical hypothyroidism was defined as mildly elevated TSH levels (5-10 mIU/L) along with normal levels of serum T4.[6] In our study, levels of TSH were higher in patients with low serum albumin and higher proteinuria. This finding can be explained by the fact that low serum albumin levels directly indicate higher urinary loss of proteins, thus higher loss of thyroid binding hormones. TSH levels are in turn increased to compensate for the urinary loss [7]. However, overt hypothyroidism that is clinically relevant was not present. Similar observations were made by Gilles et al in their study of hypothyroidism in patients of proteinuria [8]. However, they had excluded anti-TPO antibody positive patients from their study.

TSH levels were also found to be negatively correlated with glomerular filtration rate and the association was statistically significant. This result can serve as an indicator of impact of thyroid dysfunction on glomerular filtration rate as this is a known fact that hypothyroid state leads to a reduction in glomerular filtration rate [9]. Mechanisms of reduced glomerular filtration rate include decreased sensitivity to $\beta$-adrenergic stimulus and decreased renin release, along with decreased angiotensin II and impaired RAAS activity [10].

Hyponatremia was observed in 13 patients, however it was not severe enough to have clinical implications. In general, hyponatremia is the commonest electrolyte abnormality observed in hypothyroid patients and is mainly attributed to a reduction in eGFR causing diminished water delivery to the distal tubular segments. Hypokalemia was present in 6 patients but not severe enough to have clinical implications.

On histopathological examination of renal biopsy, the most common finding among the hypothyroid patients with nephrotic syndrome was membranous glomerulopathy, found in $48 \%$ of our study subjects [11-14]. The deposition of circulating immune complexes has been implicated in pathogenesis [15]. However, in our study $72 \%$ of patients with membranous nephropathy were anti-TPO antibody negative. The mechanism of autoimmunity causing this type of nephropathy could probably be explained by other antibodies not tested in this study.

There have been few studies showing the association of autoimmune hypothyroidism with various types of nephrotic syndrome. A retrospective study on 28 patients with Hashimoto's thyroiditis revealed that the most common associated kidney disease was membranous glomerulonephritis. Hashimoto's thyroiditis has also been found in patients of lupus nephritis, minimal change disease, IgA nephropathy, membranoproliferative glomerulonephritis and focal segmental glomerulosclerosis $[16,17]$. In our study, thyroid autoantibodies were positive in $41.7 \%$ of study subjects. Although there was no documented evidence of an association of anti TPO antibodies with nephrotic syndrome or any other form of renal dysfunction, a few mechanisms have been postulated for the possible association. Autoimmunity serves as the strongest predictor. Immune complex deposits have been found in basement membrane of thyroid follicular epithelium and glomeruli in some reported patients of Hashimoto thyroiditis and membranous glomerulopathy [18].

Factors supporting the autoimmune pathogenesis of renal and thyroid dysfunction include their common association with other autoimmune diseases such as type I diabetes [19]. The presence of deposits of immunoglobulins and thyroglobulin in the glomeruli of some patients is also 
an indirect indicator of common pathogenesis of both renal and thyroid dysfunction.

Glomerular disease was found in many autoimmune conditions such as lupus nephritis, ANCA associated vasculitis that can also be associated with autoimmune thyroid disease. In a study conducted by Pyne et al, the prevalence of hypothyroidism in SLE patients was greater than that of normal population [20]. This is supported by higher prevalence of Anti-TPO and anti-thyroglobulin antibodies in their study. In our study, $16 \%$ patients with positive anti-TPO antibodies had lupus nephritis.

\section{Conclusion}

Our study shows a definite correlation between nephrotic syndrome and thyroid function abnormalities. Due to major renal handling of thyroid hormones, all patients with nephrotic range proteinuria are at risk of developing clinical and subclinical hypothyroidism. Due to some overlapping features of both the diseases such as facial puffiness, weight gain and fatigue, hypothyroidism can easily be missed unless it becomes severe enough to cause gross changes in the patient's appearance and starts to impair the cardiovascular and other major organ systems of the body. Moreover, if left untreated, hypothyroidism can further impair renal functioning. Our study established a significant correlation between thyroid autoimmunity and nephrotic syndrome. Thus, a high index of suspicion should be kept in all patients with nephrotic syndrome and thyroid function tests, while tests for antibodies against thyroid antigen should be a part of routine screening in these patients.

\section{References}

1. Feinstein EI, Kaptein EM, Nicoloff JT, Massry SG. Thyroid function in patients with nephrotic syndrome and normal renal function. Am J Nephrol. 1982;2:70-76.

2. Junglee NA, Scanlon MF, Rees DA. Increasing thyroxine requirements in primary hypothyroidism: don't forget the urinalysis. J Postgrad Med. 2006;52:201-203.

3. Suher M, Koc E, Ata N, Ensari C. Relation of thyroid disfunction, thyroid autoantibodies, and renal function. Ren Fail. 2005;27:739-742.

4. Iglesias P, Díez JJ. Thyroid dysfunction and kidney disease. Eur J Endocrinol. 2009;160:503-515.
5. Hull RP, Goldsmith DJ. Nephrotic syndrome in adults. BMJ. 2008;336:1185-1189.

6. Cooper DS. Clinical practice. Subclinical hypothyroidism. N Engl J Med. 2001;345:260-265.

7. Basu G, Mohapatra A. Interactions between thyroid disorders and kidney disease. Indian J Endocrinol Metab. 2012;16:204-213. 8. Gilles R, den Heijer M, Ross AH, Sweep FC, Hermus AR, Wetzels JF. Thyroid function in patients with proteinuria. Neth J Med. 2008;66:483-485.

9. Mahjoub S, Ben Dhia N, Achour A, Zebidi A, Frih A, Elmay M. Primary hypothyroidism and glomerular involvement. Ann Endocrinol (Paris). 1991;52:289-292.

10. Allon M, Harrow A, Pasque CB, Rodriguez M. Renal sodium and water handling in hypothyroid patients: the role of renal insufficiency. J Am Soc Nephrol. 1990;1:205-210.

11. Mariani LH, Berns JS. The renal manifestations of thyroid disease. J Am Soc Nephrol. 2012;23:22-26.

12. Salomon MI, Di Scala V, Grishman E, Brener J, Churg J. Renal lesions in hypothyroidism: a study based on kidney biopsies. Metabolism. 1967;16:846-852.

13. Valentin M, Bueno B, Gutiérrez E, Martínez A, González E, Espejo B, et al. Membranoproliferative glomerulonephritis associated with autoimmune thyroiditis. Nefrologia. 2004;24 Suppl 3:43-48.

14. Grcevska L, Polenakovic M, Petrusevska G. Membranous nephropathy associated with thyroid disorders. Nephron. 2000;86:534-535.

15. Shima Y, Nakanishi K, Togawa H, Obana M, Sako M, Miyawaki M, et al. Membranous nephropathy associated with thyroid-peroxidase antigen. Pediatr Nephrol. 2009;24:605-608.

16. Santoro D, Vadalà C, Siligato R, Buemi M, Benvenga S. Autoimmune Thyroiditis and Glomerulopathies. Front Endocrinol (Lausanne). 2017;8:119.

17. Enriquez R, Sirvent AE, Amorós F, Andrada E, Cabezuelo JB, Reyes A. IgA nephropathy and autoimmune thyroiditis. Vol. 57, Clin Nephrol. 2002;57:406-407.

18. Jordan SC, Johnston WH, Bergstein JM. Immune complex glomerulonephritis mediated by thyroid antigens. Arch Pathol Lab Med. 1978;102:530-533.

19. Dizdar O, Kahraman S, Gençtoy G, Ertoy D, Arici M, Altun B, et al. Membranoproliferative glomerulonephritis associated with type 1 diabetes mellitus and Hashimoto's thyroiditis. Nephrol Dial Transplant. 2004;19:988-989.

20. Pyne D, Isenberg DA. Autoimmune thyroid disease in systemic lupus erythematosus. Ann Rheum Dis. 2002;61:70-72. 